\title{
P231: Risk factors for Acinetobacter baumannii colonization and infection among patients admitted to intensive care units
}

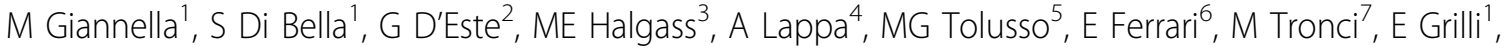 \\ N Corradetti ${ }^{8}$, N Petrosillo $^{1 *}$
}

From 2nd International Conference on Prevention and Infection Control (ICPIC 2013)

Geneva, Switzerland. 25-28 June 2013

\section{Introduction}

Active surveillance cultures (SCs) for Acinetobacter baumannii (AB) has been suggested as a strategy to control $A B$ spread in Intensive Care Units (ICUs). However, standardized screening shemes and understanding of which patients and body sites are most commonly colonized by $A B$ are lacking.

\section{Objectives}

To identify risk factors for $\mathrm{AB}$ colonization upon ICU admission.

\section{Methods}

Multicenter prospective study of all patients admitted for $\geq 48$ hours to 6 ICUs, in Rome. SCs included rectal swab (RS) and pharyngeal swab (PS) or tracheal aspirate (TA) in patients on mechanical ventilation. SCs were taken on ICU admission and once weekly until discharge from ICU.

\section{Results}

From May to Sept 2012, 847 patients were admitted to the 6 ICUs; 261 remained for $\geq 48$ hours and 201 were screened. Overall, 359 RS, 270 TA and 264 PS were taken and $\mathrm{AB}$ was isolated in $5.6 \%, 8.1 \%$ and $1.1 \%$ of them, respectively, with a total of $53 \mathrm{AB}$. All the strains were resistant to carbapenems. Of the 201 screened patients, $59.2 \%$ were male, the median age was 65 years (IQR 50-75), median Charlson and APACHE II scores were 5 (IQR 3-6) and 13 (IQR 9-17), respectively. Commonest causes for ICU admission were respiratory

'2nd Division of Infectious Diseases, National Institute of Infectious Diseases "Lazzaro Spallanzani", Italy

Full list of author information is available at the end of the article failure (32.3\%) and post surgery (30.2\%). Fourteen patients (7\%) were found to be $\mathrm{AB}$ colonized at ICU admission, whereas 9 (4.5\%) became colonized during ICU stay within a median of 7 days (IQR 6-10). Significant differences between $\mathrm{AB}$ colonized and non-colonized patients at ICU admission were found for septic shock $(21.4 \%$ vs. $4.3 \%)$ and prior antibiotic therapy (78.6\% vs. $43.3 \%)$.

\section{Conclusion}

Our preliminary analysis showed that prior antibiotic therapy and septic shock were significantly associated with $\mathrm{AB}$ colonization at ICU admission.

\section{Disclosure of interest}

None declared.

\begin{abstract}
Author details
${ }^{1}$ 2nd Division of Infectious Diseases, National Institute of Infectious Diseases "Lazzaro Spallanzani", Italy. ${ }^{2}$ Intensive Care Unit, Policlinico Casilino, Italy. ${ }^{3}$ Microbiology, Policlinico Casilino, Italy. ${ }^{4}$ Cardiosurgical Intensive Care Unit, Ospedale San Camillo, Italy. Intensive Care Unit, Ospedale San Camillo, Italy. ${ }^{6}$ Neurosurgical Intensive Care Unit, Ospedale San Camillo, Italy.

${ }^{7}$ Microbiology, Ospedale San Camillo, Italy. ${ }^{8}$ Microbiology, Aurelia Hospital, Roma, Italy.
\end{abstract}

Published: 20 June 2013

\section{doi:10.1186/2047-2994-2-S1-P231}

Cite this article as: Giannella et al.: P231: Risk factors for Acinetobacter baumannii colonization and infection among patients admitted to intensive care units. Antimicrobial Resistance and Infection Control 2013 2(Suppl 1):P231.

\section{C)

(c) 2013 Giannella et al; licensee BioMed Central Ltd. This is an Open Access article distributed under the terms of the Creative Commons Attribution License (http://creativecommons.org/licenses/by/2.0), which permits unrestricted use, distribution, and reproduction in any medium, provided the original work is properly cited. 